\title{
Untersuchungen auf dem Gebiet der Synthese von schwefelhaltigen heterozyklischen Verbindungen
}

\author{
Juri A. Moskwitschew, Jewgeni M. Alow, Aleksej W. Tarasow, Oleg P. Jablonski, Dmitri B. Kobylinski
}

Schwefelhaltige organische Verbindungen gehören zu den chemischen Verbindungen, die in verschiedenen Bereichen der Wissenschaft und Technik breite Verwendung finden. In der Staatlichen Technischen Universität Jaroslawl werden Darstellungsverfahren verschiedener substituierter Diphenylsulfonderivate und deren mehrkerniger Analoge entwickelt, die als Ausgangsstoffe für die Herstellung industrieller Polyesthersulfone, Polyamide und Polyesther, sowie von Mono- und Disulfonylchloriden, Dithiolen mit Diphenyl-Brückenbindungen, einer Reihe ungesättigter aromatischer Sulfide und Sulfone, Sulfonylaziden, Salzen der Sulfonsäuren, die ihrerseits als Monomere, vernetzende Agenzien und Zwischenprodukte bei der Synthese einer ganzen Reihe von Stoffen wie Arzneimitteln, Schaumbildnern, Anstrichstoffen, Pestiziden usw. eingesetzt werden [1-6]. Zur Zeit werden weitere Untersuchungen auf dem Gebiet der heterozyklischen und insbesondere der schwefelhaltigen Verbindungen durchgeführt.

\section{Die Synthese neuer Sulfonylchloride aus substituierten Diphenylsulfonen und deren Derivaten}

Eine ganze Reihe von Derivaten des Diphenylsulfons weisen nützliche Eigenschaften auf. So konnten bei einigen Verbindungen heilende Eigenschaften, biologische Aktivität, antiseptische, fungizide, herbizide und akarizide Eigenschaften nachgewiesen werden [10].

Der einfachste und bekannteste Vertreter der Arylensulfimide (gemischte Imide der Sulfobenzoesäuren) ist Saccharin - der erste künstliche Zuckeraustauschstoff. Das Saccharin und dessen Derivate wurden getestet und erwiesen sich als hervorragende Komponenten in Pestiziden [7], Fungiziden und Bakteriziden [8], als Zusatzstoff bei der Vernickelung [9], als Monomer bei der Herstellung hitzebeständiger Polymere [5] u.v.m.

Um Produkte synthetisieren zu können, die Fragmente dieser Verbindungen enthalten, wurde von unserer Gruppe die Gewinnung von neuen 1,1-Dioxyd-1,2-Benzisothiazolon-Derivaten auf der Grundlage der Sulfierung von Diphenylsulfonderivaten untersucht. Die Gewinnung der Ausgangsverbindungen mit Hilfe von Friedel-Krafts-Katalysatoren oder nach der Pyrosulfonatmethode sind in $[1,2,6]$ beschrieben. Die Synthese von Di- und Monosulfonsäuren der Diphenylsulfonreihe wurden in der Literatur bisher nicht behandelt. Eine Ausnahme bilden die Disulfonylchloride aus symmetri- schen Diphenylsulfonderivaten, die durch einen Überschuß von Chlorsulfonsäure dargestellt werden und bei der Synthese von wärmebeständigen Polykondensationspolymeren Verwendung finden [11]<smiles>[R]c1ccc(S(=O)(=O)c2ccc([R])cc2)cc1</smiles>
1

wobei $\mathrm{R}=\mathrm{H}, \mathrm{CH}_{3}, \mathrm{Cl}$ sind.

Wir haben einige kinetische Gesetzmäßigkeiten der Sulfierung von Verbindungen [1] durch verschiedene Sulfierungsagenzien unter gleichen Bedingungen untersucht. Die Reaktionsgeschwindigkeit der Sulfierung von Sulfonen (I) war stark abhängig vom Substituenten R (max. Faktor 1000). Das bietet die Möglichkeit, die Synthese von bereits bekannten Sulfonylchloriden (II) sowie die selektive Gewinnung von bisher unbekannten Monosulfonsäuren und der entsprechenden Sulfonylchloriden auf der Basis zugänglicher Diphenylsulfonderivate und Oleum oder der Chlorsulfonsäure nach dem folgenden Schema zu optimieren,

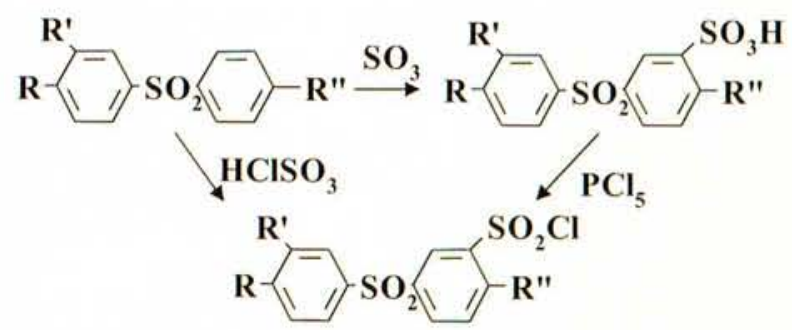

wobei $\mathrm{R}=\mathrm{H}, \mathrm{CH}_{3}, \mathrm{Cl}, \mathrm{Br}, \mathrm{NO}_{2}, \mathrm{COOH} ; \mathrm{Rc}=\mathrm{H}, \mathrm{Cl} ; \mathrm{Rcc}=$ $\mathrm{H}, \mathrm{CH}_{3}, \mathrm{Cl}, \mathrm{Br}, \mathrm{NO}_{2}, \mathrm{COOH}$ sind. Dasselbe gilt für die selektive Gewinnung der Disulfonchloride von dem Diphenylsulfon nach dem Schema:

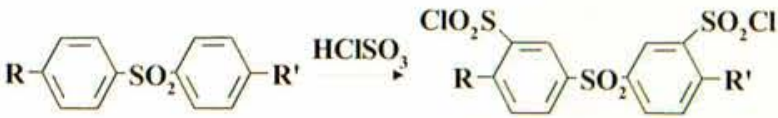

wobei $\mathrm{R}=\mathrm{CH}_{3} ; \mathrm{R}^{\prime}=\mathrm{H}, \mathrm{Cl}, \mathrm{Br}, \mathrm{OCH}_{3}$ sind.

Entscheidend bei der Auswahl der Sulfierungsagenz und der Reaktionsbedingungen (Temperatur, Zeit und Molverhältnis der Reagenzien) sind das Wesen und der absolute Wert der $\sigma$-Konstanten der Substituenten, die in verschiedenen Diphenylsulfonkernen vorhanden sind. Durch Ammonolyse einer Reihe der dargestellten Sulfochloride und anschließender Oxydierung mit Natrium- 
bichromat wurden neue Saccharine synthetisiert,

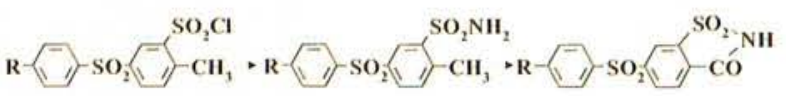

wobei $\mathrm{R}=\mathrm{H}, \mathrm{CH}_{3}, \mathrm{Cl}, \mathrm{Br}, \mathrm{NO}_{2}, \mathrm{COOH}$ sind.

Mit Hilfe des gleichen Verfahrens wurden Saccharine aus Disulfochloriden hergestellt,

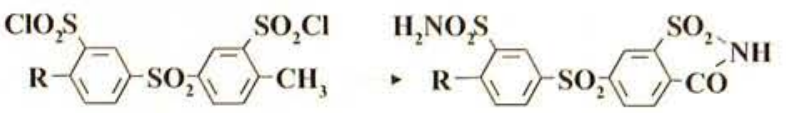

wobei $\mathrm{R}=\mathrm{H}, \mathrm{Cl}, \mathrm{Br}, \mathrm{OCH}_{3}$ sind.

Im Falle von $\mathrm{R}=\mathrm{CH}_{3}$ wurde in der letzten Reaktion das symmetrische 6,6'- bis (1,1-Dioxyd-1,2-Benzisothiazolon) Sulfon dargestellt.

Die aus 1,1-Dioxyd-Benzisothiazolon neu synthetisierten Diphenylsulfonderivate, deren Struktur durch chemische und spektrale Methoden nachgewiesen wurde, können nun beispielsweise als Ausgangsstoffe für die Herstellung von wärmebeständigen Polysulfimiden, als Zusatzstoffe beim Vernickeln sowie als Ausgangsstoffe bei der Herstellung von Herbiziden und Pfanzenwachstumsmitteln genutzt werden.

\section{Die Synthese neuer 1,3,4-Thiadiazolderivate}

1,3,4-Thiadiazolderivate finden breite Verwendung als pharmazeutische Präparate, Oxydationsinhibitoren, komplexbildende Reagenzien u. a. |12-15]. Auf deren Grundlage wurden ebenso hochhitzebeständige Polymere mit wertvollen mechanischen Eigenschaften dargestellt.

Im Zusammenhang mit dem Interesse an 1,3,4-Thiadiazolderivaten synthetisierten wir einige neue 1,3,4-Thiadiazole, um deren biologische Aktivität zu untersuchen. Es ist bekannt, daß als geeignete Synthone für die Synthese von 2-Amin-5-substituierten 1,3,4-Thiadiazolen 1Acetylthiosemicarbazide dienen [16]. In dieser Arbeit wurden von uns 1-Arenthio- und 1-Arensulfonylacetylthiosemicarbazide (III) als Ausgangsstoffe verwendet, die ihrerseits aus den Hydraziden der Arenthio- und Arensulfonylessigsäuren und Propionsäuren synthetisiert worden sind.

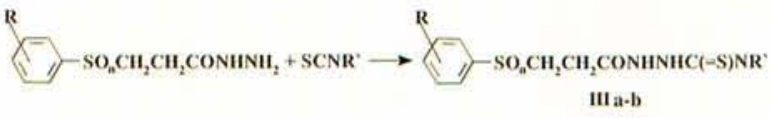

wo $\mathrm{R}=\mathrm{CH}_{3}, \mathrm{Br}, \mathrm{Cl} ; \mathrm{R}^{\prime}=\mathrm{H}, \mathrm{Ph} ; \mathrm{a}: \mathrm{n}=0 ; \mathrm{b}: \mathrm{n}=2$

Die Synthese (IIla-b, R' = H) wurde durch die Reaktion zwischen Hydrazid und Kaliumrhodanid im wäßrigen Medium in Anwesenheit von Salzsäure durchgefuihrt. Die Reaktion zwischen dem Hydrazid und Phenylisothiocianat in 1,4-Dioxan führt zu 1-Acetylthiosemicarbazid (IIla-b, $\left.\mathrm{R}^{\prime}=\mathrm{Ph}\right)$.
Es konnte gezeigt werden, daß durch kurze Erwärmung der Reagenzien (III) in Anwesenheit von konzentrierter $\mathrm{H}_{2} \mathrm{SO}_{4}$ oder Polyphosphorsäure die Heterozyklisierung initiiert wird. Es bilden sich 2-Amin-5- substituierte 1,3,4-Thiadiazole mit einer Ausbeute von 80 bis $90 \%$.

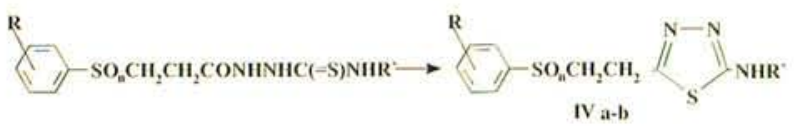

In den IR- Spektren der Verbindungen (IVa-b) sind Absorptionsbanden im Bereich 1520, 1490, 1380, 1230, 1050 und $860 \mathrm{~cm}^{-1} \mathrm{zu}$ beobachten, die für die Schwingungen des 1,3,4-Thiadiazolrings typisch sind, sowie Absorptionsbanden im Bereich von $1320-1300 \mathrm{~cm}^{-1}$, die den Valenzschwingungen der $\mathrm{C}-\mathrm{N}$-Bindung in Aminen zugeordnet werden können [17].

In PMR- Spektren von 2-Amin-5- substituierten 1,3,4Thiadiazolen (IVa-b) sind Protonensignale der aromatischen Kerne (7,1-8,5 ppm) und zwei Tripletts im Bereich von 3,9 und $3,7 \mathrm{ppm}\left(-\mathrm{CH}_{2} \mathrm{CH}_{2}^{-}\right)$vorhanden.

Hervorzuheben ist, daß bei der Heterozyklisierung der Verbindungen (III) mit $\mathrm{R}^{\mathrm{\prime}}=\mathrm{H}$ die Ausbeute im $\mathrm{H}_{2} \mathrm{SO}_{4}{ }^{-}$ Medium etwas niedriger ( 80 bis $85 \%$ ) als bei der Zyklisierung in Polyphosphorsäure (90 bis $95 \%$ ) war. In $\mathrm{H}_{2} \mathrm{SO}_{4}$ konnte außerdem eine starke Harzbildung beobachtet werden. Aus diesem Grund haben wir als Reaktionsmedium die Polyphosphorsäure gewählt.

Durch die Azylierung der Verbindungen (IV) mit Chloranhydriden der Sulfon- und Carbonsäuren bei Tertiäraminen wurden entsprechende Amide (Va-b) und Sulfamide (Vla-b) synthesiert,

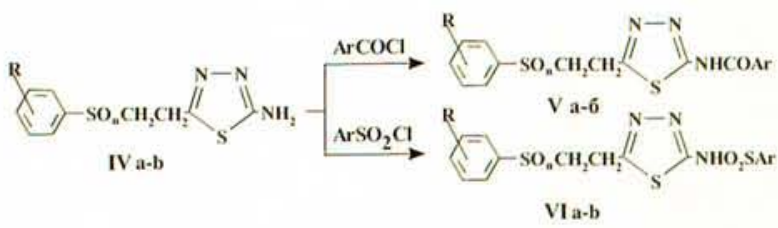

wobei $\mathrm{Ar}=4-\mathrm{ClC}_{6} \mathrm{H}_{4}, 4-\mathrm{CH}_{3} \mathrm{C}_{6} \mathrm{H}_{4}, 4-\mathrm{BrC}_{6} \mathrm{H}_{4}$ u. a. sind.

Die Azylierung wurde in 1,4- Dioxan oder DMAA durchgeführt. Die Ausbeute der Verbindungen (V, VI) betrug 75 bis $96 \%$.

Die Synthese von Amiden wurde außerdem durch Heterozyklisierung von 1,4-Diacetylthiosemicarbazid durchgefuihrt.

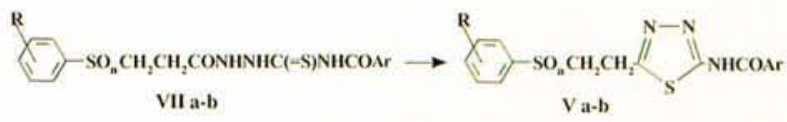

Es konnte gezeigt werden, daß bei Erwärmung des 1,4Diacetylthiosemicarbazid in Eisessigsäure die Zyklisierungsreaktion leicht abläuft und sich Amide der 5- substituierten 1,3,4-Thiadiazolen (V) mit einer Ausbeute von etwa $90 \%$ bilden. In den Reaktionsprodukten konnten keine anderen heterozyklischen Verbindungen nachgewiesen werden. 
Die IR-Spektren der Verbindungen VI haben Absorptionsbanden bei $1340-1330 \mathrm{~cm}^{-1}$ und $1160-1150 \mathrm{~cm}^{-1}$, die wir den asymetrischen valenten Schwingungen der $-\mathrm{SO}_{2}$-Gruppe in Sulfamiden zugeschrieben haben. Für Amidderivate (V) ist das Vorhandensein vom Banden im Bereich von 1650-1620 $\mathrm{cm}^{-1}$ typisch.

In PMR-Spektren der Verbindungen (V,VI) sind im Bereich von 10-11 ppm Protonensignale als Singulett vorhanden, die den primären Amid- und Sulfamidgruppen entsprechen.

Mit Metallsalzen bilden 2-Amin-5-substituierte 1,3,4Thiadiazole farbige Niederschläge: $m i t \mathrm{Cu}^{2+}$ - dunkelgriine, mit $\mathrm{Ni}^{2+}$ - dunkelblaue, mit $\mathrm{Fe}^{2+}$ - braune, mit $\mathrm{Zn}^{2+}$ weiße.

\section{Literatur}

[1] Moskwitschjow J. A., Kramerova S. K., Belyschewa M. S., Mironov G. S., Alov Je. M. u. a. (UdSSR). Shurnal prikladnoi chimii. 1980. T. 53, N3. S. 706-709. (russ) /Zeitschrift für angewandte Chemie/.

[2] Moskwitschjow J. A., Schapiro Ju. Je., Timoschenko G. N., Gerasimowa N. P. (UdSSR). Shurnal organitscheskoi chimii. 1984. T. 20, N5. S. 1032-1037. (russ) /Zeitschrift für organische Chemie/.

[3] Sergejew W. A., Nedelkin W. I., Astankov A. W., Moskwitschjow J. A., Alov Je. M., Nikiforow A. W. (UdSSR). Iswestija Akademii nauk SSSR. Ser. Chim. 1990. N4. S. 863867. (russ) /Nachrichten der Akademie der Wissenschaften der UdSSR/.

[4] Amosowa S. W., Anziferowa L. I., Schaulina L. P., Golentkowskaja I. P., Nosyrewa W. W., Achramowitsch O. Je., Kim-Pi-I O. T., Tarasow A. W., Moskwitschjow J. A. (Rußland) Shurnal prikladnoi chimii. 1996. T. 69, N8. S. 13001303. (russ) /Zeitschrift für angewandte Chemie/.

[5] Gaza Je. B., Moskwitschjow J. A., Krjukowa G. G., Mironow G. S., Farberov M. I. (UdSSR). Shurnal organitscheskoi chimii. 1974. -T.10, N8. -S. 1793-1797. (russ) /Zeitschrift für organische Chemie/.

[6] Mironow G. S., Moskwitschjow J. A., Farberov M. I. Phosphor and sulfur. 1979. V.6. N1-2. P. 209-210.

[7] Pat. USA 5723414. Int. Cl. C07D 417/06. 5-Hydroxypyrazol-4-ylcarbonyl-substituted saccharin derivates.

[8] Pat. USA 47133389. Int. Cl. C07D 275/06. Fungicidalli and bactericidally active acylated saccharin derivates.

[9] Pat. USA 4661216. Int. Cl. C07D 234/94. Elektrodepositing Co-Ni-Fe alloys for film heads.

[10] Melnikow N. N. (UdSSR). Chimija i technologija pestizidow/M.: Chimija, 1974. 766 s. (russ)/Chemie und Technologie von Pestiziden Moskau: Chemie/.

[11] Bjuller K. L. (UdSSR). Teplo- i termostoikije polimery. Per. s nem.: pod red. Wygodskogo Ja. S. M. Chimija, 1984, 1056 s. (russ) /Wärme-und Thermobeständige Polymere. Übersetzung aus dem Deutschen: Bei Redakion von Wygodski Ja. S. Moskau: Chemie/.

[12] Postowski I. Ja., Weretschschagina N. N. (UdSSR). Shurnal obtschschei chimii. 1956. T. 26, wyp.1. S. 2583-2588. (russ) /Zeitschrift für allgemeine Chemie/.

[13] Gaca J., Rauckyte T., Zac S., Szramka R. (Polen) XL Zjazd naukovy. Polskiego towarzystwa chemicznego i stowarzyszenia inzynierow i technikow przemyslu chemicznego: Streszczenia. Gdansk. 1997. S. 6. P-8. (polnisch).
[14] White A. D., Creswell M. W., Chucholowski O. W. J. Med. Chem.- 1996. V.39, N 22. S. 4382-4395.

[15] Pat. 3887572 USA, Int Cl. CO7D 93/62. Preparation of 2 amino-5-alkyl-1,3,4-thiadiayols.

[16] T. Dshilkrist. (Rußland) Chimija geteroziklitcheskich sojedineni / Per. s angl.: Pod. red. Jurowskoi M. A.: Mir; 1996. S. 464. (russ.)/Chemie von heterozyklischen Verbindungen/Übersetzung aus dem Englischen: Bei Redaktion von Jurowskaia M. A. Moskau: Mir/.

[17] Fisitscheskije metody w chimii geteroziklitcheskich sojedineni / Pod. red. A. R.Katriykogo. M.: Mir, 1966. S. 515. (russ.) / Physikalische Methoden in der Chemie von Heteroziklischen Verbindungen / Bei Redaktion von A. P. Katrizki. - Moskau, Leningrad: Mir\%.

\section{Verfasser}

Prof. Dr. habil. Juri Aleksandrowitsch Moskwitschew Rektor der Jaroslawler Staatlichen Technischen Universität

Leiter des Lehrstuhls für Technologie der organischen Stoffe und

Mitglied der Russischen Akademie für Wissenschaften

Prof. Dr. habil. Jewgeni Michailowitsch Alow

Leiter des Lehrstuhls für organische Chemie

\section{Dr. Aleksej Walerjewitsch Tarassow}

Lehrstuhl für Technologie der organischen Stoffe

Prof. Dr. habil. Oleg Pawlowitsch Jablonski

Leiter des Lehrstuhls für analytische Chemie

\section{Dmitri Borissowitsch Kobylinski}

Doktorand am Lehrstuhl für Technologie der organischen Stoffe

Staatliche Technische Universität Jaroslawl Moskowskij Prospekt 88, RUS-Jaroslawl 150023

Tel. (007 852) 441519 und 441230

Fax (007 852) 440729

Übersetzung:

Dr. Olga Rösch

Leiterin des Sprachenzentrums der

Technischen Fachhochschule Wildau 